Bulyk R. Ye., Smetaniuk O. V., Bulyk T. S., Kryvchanska M. I. Morphometric analysis of supraoptic neurons of the rat hypothalamic nuclei under conditions of prolonged illumination. Journal of Education, Health and Sport. 2021;11(10):215-220. eISSN 2391-8306. DOI http://dx.doi.org/10.12775/JEHS.2021.11.10.019

https://apcz.umk.pl/JEHS/article/view/JEHS.2021.11.10.019

https://zenodo.org/record/5619986

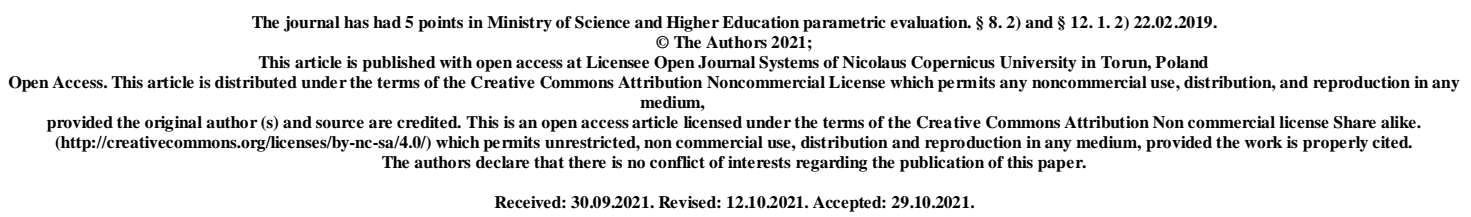

UDC 591.185.6

\title{
MORPHOMETRIC ANALYSIS OF SUPRAOPTIC NEURONS OF THE RAT HYPOTHALAMIC NUCLEI UNDER CONDITIONS OF PROLONGED ILLUMINATION
}

\author{
R. Ye. Bulyk, O. V. Smetaniuk, T. S. Bulyk, M. I. Kryvchanska
}

Bukovynian State Medical University, Chernivtsi, Ukraine

R.Ye.Bulyk, Head of the Department of Medical Biology and Genetics, Bukovynian State Medical University, M.D., Full Professor, Chernivtsi, Ukraine

O.V.Smetaniuk, Assistant Professor of the Department of Medical Biology and Genetics, Bukovynian State Medical University, Chernivtsi, Ukraine

T.S.Bulyk, Assistant Professor of the Department of Obstetrics and Gynecology, Bukovynian State Medical University, PhD Medicine, Chernivtsi, Ukraine

M.I.Kryvchanska Assistant Professor of the Department of Medical Biology and Genetics,

Bukovynian State Medical University, PhD Medicine, Chernivtsi, Ukraine

\section{Abstract}

The article reviews the results of studies of the morphofunctional state of neurons of the supraoptic nuclei of the rat hypothalamus under conditions of different duration of light regime. Under standard light regime in rats, a diurnal rhythm of morphofunctional activity of supraoptic nucleus neurons with maximum activity during daytime (before 2 p.m.) is recorded.

In animals subjected to prolonged light exposure, more pronounced changes in the morphofunctional state of the supraoptic neurons of the hypothalamus at 2 a.m. than at 2 p.m. were established. Thus, the neuronal nucleus area was $94.08 \pm 9.55 \mu \mathrm{m}^{2}$ and was significantly 
greater than that in intact animals. The nucleo-cytoplasmic ratio of supraoptic hypothalamic neuron at 2 a.m. was lower than that in intact animals due to a decrease in specific nucleus volume. In comparison with the day period ( 2 p.m.), before 2 a.m. there was revealed a decrease of the neuron body area of supraoptic nuclei of hypothalamus due to possible decrease of the area of nucleus and nucleolus of cells. This was the reason for the increase in the nucleo-cytoplasmic ratio in the neurons under observation at night, which was $2.51 \pm$ 0.023 units. Constant light regime did not cause inversion of the rhythm of morphofunctional activity of the neurons under study, the maximum values, as in intact animals, occurred in the daytime observation period.

Key words: supraoptic nucleus; morphofunctional state; constant illumination.

Relevance of the study. Many physiological and behavioral processes manifest circadian rhythms generated by internal chronometric systems, biological clock [1, 4]. In mammals the location of main pacesmaker, which controls circadian rhythms, is suprachiasmatic nucleus of hypothalamus [6]. Synchronization of the pacemaker by geophysical diurnal cycles occurs by means of illumination $[2,10]$. From the suprachiasmatic nucleus of hypothalamus, information about illumination spreads to the pineal gland (brain epiphysis) [2,9]. The gland is a part of the system, which is able to perceive changes in the ambient light level and ensure circadian rhythms of the body functioning, in particular by synthesizing its leading indole, melatonin $[1,5]$. It has been shown that melatonin secretion is subject to distinct diurnal variations with a minimum value in the daytime and a maximum around 2 a.m. [8]. Irregularity in the light regime (prolonged light, constant darkness) is a defining stressor, which leads to the development of desynchronosis [7].

One of the important links of the hypothalamic neuroendocrine system is the supraoptic nuclei, whose neurons synthesize vasopressin and oxytocin, transport them to the neurohypophysis and subsequently to the bloodstream [11]. Suprachiasmatic nuclei are also involved in providing the neuroendocrine response to various types of stress, immobilization, traumatic, emotional, pain, hypoxic, light and stresses of other etiologies.

Mechanisms of circadian pacing activity of neuronal systems of hypothalamic suprachiasmatic nuclei are currently subjected to intensive research. At the same time, the information concerning the effects of photoperiod modifications (in particular, constant light) on the activity of the most vulnerable hypothalamic structures (supraoptic nuclei), which ensure the sequence of neuroendocrine changes under stress, and the stress-responsiveness of the organism, remains relatively limited. 
The aim of the study was to clarify the effect of light stress on the morphofunctional state of the supraoptic nuclei of the rat hypothalamus.

Materials and methods. Experimental animals were divided into 2 study series, in each of which the biomaterial was taken at 2 p.m. and at 2 a.m. The chosen terms of the experiment were determined by different functional activity of the pineal gland at the given time periods of the day.

The animals of the first series (intact) were kept for 7 days under conditions of usual light regime (light from 8 a.m. to 8 p.m., illumination by fluorescent lamps of 500 lux at the cage level). Rats of the second series were kept under conditions of constant light (simulation of pineal gland hypofunction) for 7 days.

Morphometric analysis of hypothalamic neurons was performed using a computer system for digital image analysis VIDAS-386 (Kontron Elektronik, Germany) in the visible spectrum. The image obtained with an AXIOSKOP microscope using a COHU-4922 video camera (COHU Inc., USA) was input into the VIDAS-386 computer system for digital image analysis (Kontron Elektronik, Germany). Image analysis was performed in semi-automated mode using VIDAS-2.5 application software package (Kontron Elektronik, Germany): neuron body limits, its nuclei and nucleoli were determined interactively.

The experimental data were processed on personal computers using VIDAS-2.5 (Kontron Elektronik, Germany) and EXCEL-2003 (Microsoft Corp., USA). For all indicators we calculated the value of the arithmetic mean of the sample (x), its dispersion, and the error of the mean $(\mathrm{Sx})$. To determine the reliability of differences between the results of the studies in the experimental and control groups of animals we determined Student's coefficient (t), after which we found the probability of difference between the samples (p) and the confidence limit for the mean according to Student's distribution tables. Values for which $\mathrm{p}<0.05$ were considered significant.

Results of the study and their discussion. The study of morphometric characteristics of the neurons of the hypothalamic supraoptic nuclei revealed that indices have the diurnal dynamics. Under the standard light regime in rats, a diurnal rhythm of morphofunctional activity of the neurons of the hypothalamic supraoptic nuclei with maximum activity in the daytime (before 2 p.m.) was registered.

It is known that among the external geophysical factors, illumination has a significant influence on the work of the circadian pacemaker. When animals were kept under conditions of constant illumination at 2 p.m., the area of the supraoptic neuron nuclei of the hypothalamus was close to the analogous value in intact rats. At the same time, we detected 
an increase in the size of its nucleus by $17.9 \pm 2.1 \%(\mathrm{r}=0.79)$. Changes in the size of the nucleus were caused by an increase in the area of the neuron nucleolus $(r=0.89)$, which was $68.13 \pm 8.97 \mu \mathrm{m}^{2}$ and was almost twice as large as that in the intact rats. The significant decrease in the nucleo-cytoplasmic ratio (NCR) in intact animals by $17.2 \pm 1.3 \%$, which was $2.41 \pm 0.030$ units, was also noteworthy. At the same time, the specific volume of the nucleolus was within $11.11 \pm 1.523$ units and was significantly higher than the volume of the studied structure in the neuron of intact animals during the daytime observation period.

Light stress led at 2 p.m. to a significant decrease in RNA concentration in the nucleus by $18.7 \pm 1.5 \%$, while in the nucleolus and cytoplasm the amount of RNA was probably greater from the values of intact animals.

Keeping animals under hyperilluminated conditions caused more pronounced changes in the morphofunctional state of the supraoptic hypothalamic nuclei neurons at 2 a.m. than at 2 p.m. Thus, the neuronal nucleus area was $94.08 \pm 9.55 \mu \mathrm{m}^{2}$ and was significantly greater than that in intact animals. The indicated changes were accompanied by a twofold increase in nucleolus area $(r=0.91)$. At the same time, the cytoplasmic area of the neuron was $200.82 \pm$ $9.071 \mu \mathrm{m}^{2}$ and was close to that in animals kept under the standard light regime. Note that the animals' stay under hyperillumination conditions did not affect the diurnal rhythm of morphofunctional activity of the supraoptic hypothalamic nuclei neurons. Their greater activity, as well as in the animals under standard illumination, was recorded during the daytime observation period.

NCR of the neuron of supraoptic hypothalamic nuclei at 2 a.m. was $4.2 \pm 0.24 \%$ lower than that in intact animals due to a decrease in the specific volume of the nucleus.

Characterizing the nocturnal stage of the experiment, we note that, like in the animals kept under the normal photoperiod, the high RNA concentration in the neurons of the supraoptic nuclei of the hypothalamus was also registered at 2 p.m. We revealed a direct correlation between RNA concentration in the nucleus and nucleus area $(r=0.32)$, RNA concentration in the nucleolus and nucleolus area $(r=0.29)$, RNA concentration in cytoplasm and area of the latter $(r=0.75)$. In this diurnal interval, the nucleic acid concentration indices in the components of the structures under study were higher as compared to the values of intact animals of the same time interval.

Compared with the daytime period (2 p.m.), a decrease in the nucleus and nucleolus area of the neuron of supraoptic hypothalamic nuclei was revealed before 2 a.m. $(r=0.89)$. This was the reason for the increase in the NCR in the studied neurons during the night observation period, which amounted to $2.51 \pm 0.023$ units. 
Conclusions. 1. The duration of the photoperiod significantly affects the daily activity of the supraoptic nuclei of the rat hypothalamus.

2. Constant light regime does not cause inversion of the rhythm of morphofunctional activity of the studied neurons, the maximum values, as in intact animals, fall on the daytime interval.

3. Light stress causes a significant increase in nucleus and nucleolus area of neurons, at night time of observation. At the same time, we observed a decrease in the nucleocytoplasmic ratio and an increase in RNA concentration in the nucleus and cytoplasm of neuronal supraoptic nuclei of rat hypothalamus during the daytime.

Prospects for further studies. In the future, we plan to study the effect of synthetic peptides of the pineal gland on the morphofunctional activity of neurons of the hypothalamic supraoptic nuclei in order to gain more insight into the mechanisms of involvement of these structures in regulation of neuroendocrine processes under stress and stress response depending on the duration of the photoperiod.

\section{References}

1. Karachentsev YuI, Kravchun NA, editors. The pineal gland and the hypothalamicpituitary-thyroid system: age and chronobiological aspects. Kharkov; 2013. 262 p.

2. Timofei OV, Bulyk RYe, Lomakina YuV. Melatonin's effects on the c-Fos gene expression in neurons of the medial small subnucleus of hypothalamus paraventricular nucleus of rats under altered light condition. World of Medicine and Biology. 2015;2(50):188-92.

3. Bedont JL, Blackshaw S. Constructing the suprachiasmatic nucleus: a watchmaker's perspective on the central clockworks. Front Syst Neurosci. 2015;9:74. doi: $\underline{10.3389 / \text { fnsys.2015.00074 }}$

4. Zamorskiy II, Sopova IYu, Khavinson VKh. The effect of melatonin and epithalamin on the content of protein and lipid peroxidation products in the cerebral cortex and the hippocampus of rat brain in acute hypoxia. Bulletin of Experimental Biology and Medicine. 2012;154(7):59-61.

5. Venegas C, García JA, Escames G, Ortiz F, López A, Doerrier C, et al. Extrapineal melatonin: analysis of its subcellular distribution and daily fluctuations. J Pineal Res. 2012;52(2):217-27. doi: 10.1111/j.1600-079X.2011.00931.X

6. Kiessling S, Sollars PJ, Pickard GE. Light stimulates the mouse adrenal through a retinohypothalamic pathway independent of an effect on the clock in the suprachiasmatic nucleus. PLoS One. 2014[cited 2021 Jun 10];9(3):e92959. doi: 10.1371/journal.pone.0092959 
7. Arushanian EB, Schetinin EV. Melatonin as a universal modulator of any pathological processes. Pathological Physiology and Experimental Therapy. 2016;60(1):7988.

8. Khavinson VKh, Linkova NS, Kvetnoy IM, Kvetnaya TV, Polyakova VO, Korf Kh. Molecular-cellular mechanisms of peptide regulation of melatonin synthesis in pinealocyte culture. Bulletin of Experimental Biology and Medicine. 2012;153(2):223-6.

9. Fernandez F, Lu D, Ha P, Costacurta P, Chavez R, Heller HC, et al. Circadian rhythm. Dysrhythmia in the suprachiasmatic nucleus inhibits memory processing. Science. 2014;346(6211):854-7. doi: 10.1126/science.1259652

10. Bedont JL, Newman EA, Blackshaw S. Patterning, specification, and differentiation in the developing hypothalamus. Wiley Interdiscip Rev Dev Biol. 2015;4(5):445-68. doi: 10.1002/wdev.187

11. Wang JL, Lim AS, Chiang WY, Hsieh WH, Lo MT, Schneider JA, et al. Suprachiasmatic neuron numbers and rest-activity circadian rhythms in older humans. Ann Neurol. 2015;78(2):317-22. doi: 10.1002/ana.24432 